Supporting Information for

\title{
Predicted Confinement-Enhanced Stability and Extraordinary Mechanical Properties for Carbon Nanotube Wrapped Chains of Linear Carbon
}

Enlai $\mathrm{Gao}^{1^{*}}$, Ruishan $\mathrm{Li}^{1}$, and Ray H. Baughman ${ }^{2 *}$

${ }^{1}$ Department of Engineering Mechanics, School of Civil Engineering, Wuhan University, Wuhan, Hubei 430072, China.

${ }^{2}$ Alan G. MacDiarmid NanoTech Institute, The University of Texas at Dallas, Richardson, TX 75080, USA.

*Corresponding authors:

Emails: enlaigao@whu.edu.cn (E.G.); ray.baughman@utdallas.edu (R.H.B.)

The Supporting Information contains

- Figures S1-S3.

- Tables S1-S3.

- Movies S1-S2. 
reactions between NTWCs and near-ambient molecules

- - - - reactions between SWNT sheath and carbyne chains

- - - - inter-carbyne-chain crosslinking reactions and solid-state polymerization

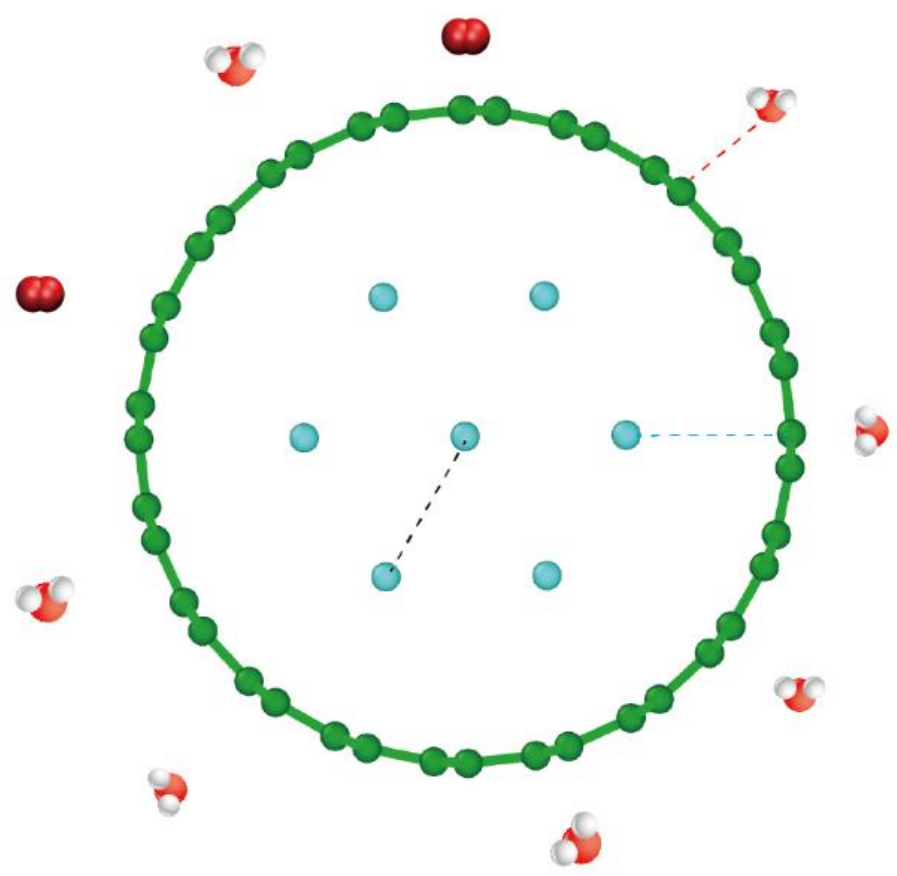

Figure S1. Possible chemical instabilities of NTWCs: (1) Reactions between NTWC and surrounding molecules, (2) reactions between the carbyne chains and the SWNT sheath, and (3) inter-carbyne-chain reactions. 

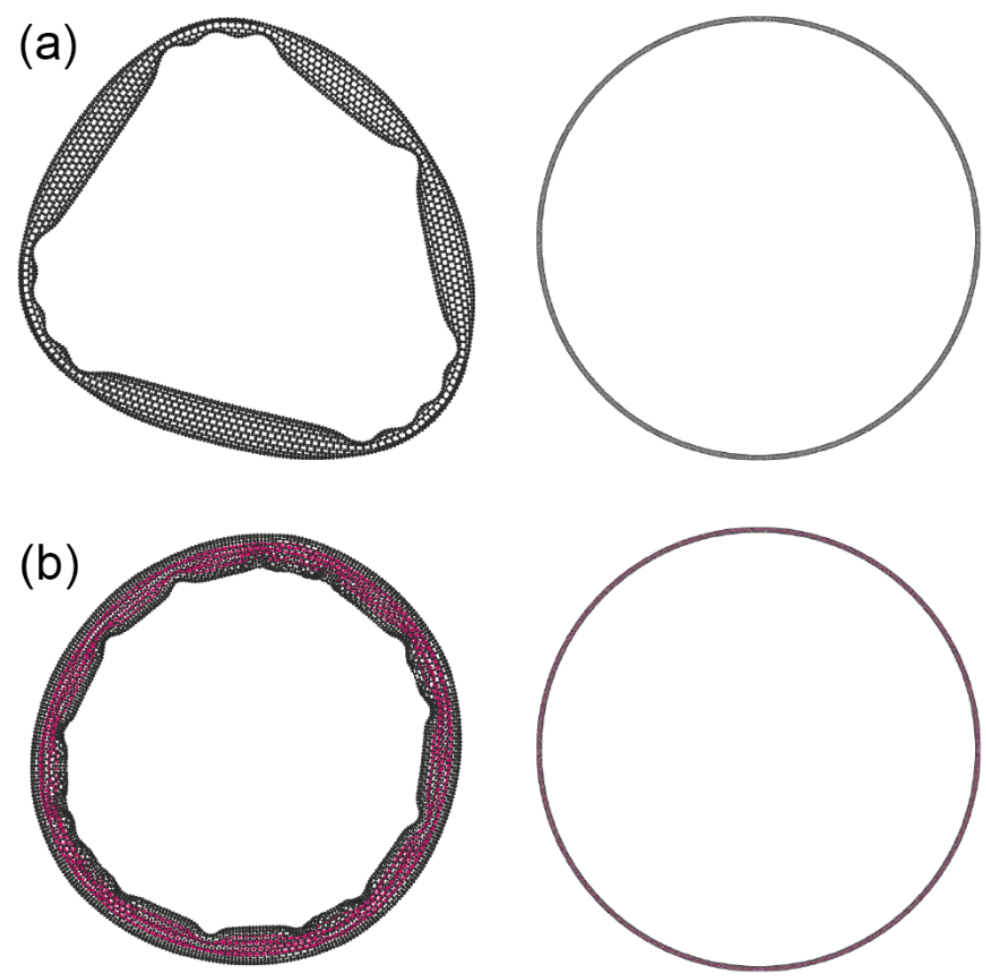

Figure S2. (a) Bending tests of (10,10) SWNT and (b) 10@7 NTWC with different radii. 


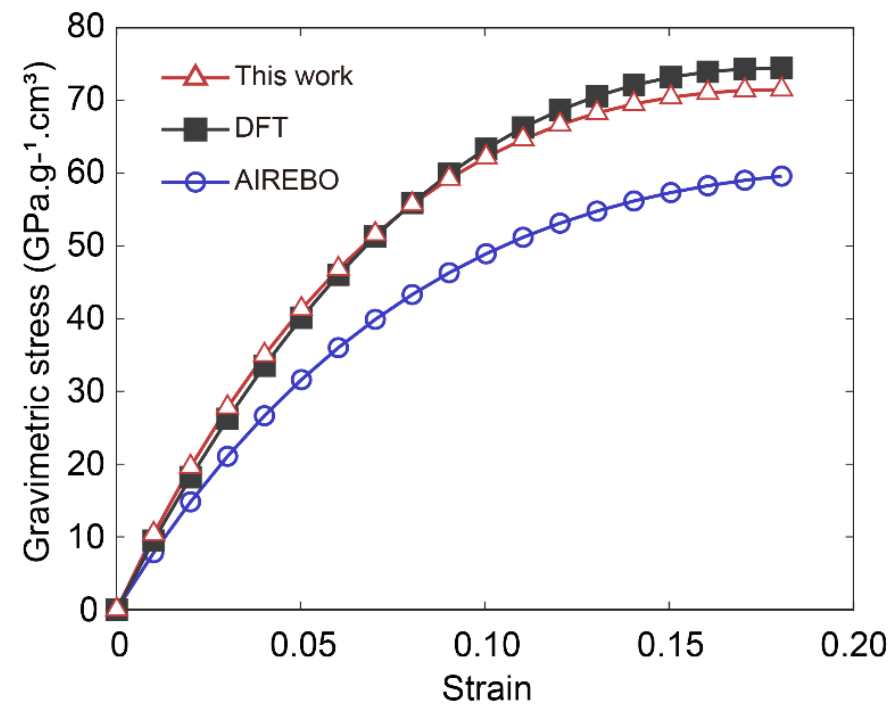

Figure S3. Gravimetric stress-strain curves of carbyne chains using the Morse potential, AIREBO potential, and DFT calculations. 
Table S1. Mechanical properties of carbyne chains calculated using different methods.

\begin{tabular}{llll}
\hline & DFT & Morse & AIREBO \\
\hline$\sigma_{\mathrm{gs}}\left(\mathrm{GPa} \cdot \mathrm{g}^{-1} \cdot \mathrm{cm}^{3}\right)$ & 74.20 & 71.20 & 60.25 \\
$Y_{\mathrm{g}}\left(\mathrm{GPa} \cdot \mathrm{g}^{-1} \cdot \mathrm{cm}^{3}\right)$ & 906.8 & 977.2 & 738.4 \\
$\varepsilon_{\mathrm{S}}(\%)$ & 18 & 18 & 22 \\
\hline
\end{tabular}

*A unit cell of the carbyne chain containing 2 atoms with periodic boundary condition along the axial direction was adopted in DTF calculations. 
Table S2. Mechanical properties of graphene calculated using different methods.

\begin{tabular}{lll}
\hline & AIREBO & DFT \\
\hline$\sigma_{\mathrm{gs}}\left(\mathrm{GPa} \cdot \mathrm{g}^{-1} \cdot \mathrm{cm}^{3}\right)$ & 50.25 & 49.01 \\
$Y_{\mathrm{g}}\left(\mathrm{GPa} \cdot \mathrm{g}^{-1} \cdot \mathrm{cm}^{3}\right)$ & 356.4 & 429.1 \\
$\varepsilon_{\mathrm{s}}(\%)$ & 30 & 24 \\
\hline
\end{tabular}

${ }^{*}$ Graphene was stretched along the zigzag direction, which is consistent with the stretching direction of the armchair SWNTs used in this work. 
Table S3. Morse-form potential developed for carbyne chains.

\begin{tabular}{lll}
\hline Interaction terms & Functional forms & Parameters \\
\hline Bond stretching & $V_{\mathrm{b}}=D\left[1-\mathrm{e}^{(-\alpha(r-\mathrm{r})}\right)^{2}$, & $D=4.6 \mathrm{eV} ; \alpha=3.0 \AA^{-1}$ \\
& with a cut off of bond length $\left(r<r_{\mathrm{c}}\right)$. & $r_{0}=1.283 \AA ; r_{\mathrm{c}}=1.514 \AA$ \\
Angle bending & $V_{\mathrm{a}}=k_{\mathrm{a}}\left(\theta-\theta_{0}\right)^{2}$ & $k_{\mathrm{a}}=1.39 \mathrm{eV} ; \theta_{0}=180^{\circ}$ \\
Non-bond & $V_{i j}^{L J}\left(r_{i j}\right)=4 \epsilon\left[\left(\frac{\sigma_{0}}{r_{i j}}\right)^{12}-\left(\frac{\sigma_{0}}{r_{i j}}\right)^{6}\right]$ & $\varepsilon=0.00284 \mathrm{eV} ; \sigma_{0}=3.4 \AA$ \\
interaction & & \\
\hline
\end{tabular}


Movie S1: Inter-carbyne-chain crosslinking reactions in free space.

Movie S2: Inter-carbyne-chain crosslinking reactions in confined space. 